\title{
A set-up for simultaneous measurement of second harmonic generation and streaming potential and some test applications
}

Johannes Lützenkirchen ${ }^{1}$, Tim Scharnweber ${ }^{2}$, Tuan $\mathrm{Ho}^{3}$, Alberto Striolo ${ }^{4}$, Marialore Sulpizi ${ }^{5}$, Ahmed Abdelmonem ${ }^{6, *}$

${ }^{1}$ Karlsruher Institut für Technologie (KIT), Institut für Nukleare Entsorgung - INE, Hermann-vonHelmholtz-Platz 1, 76344 Eggenstein-Leopoldshafen, Germany, johannes.luetzenkirchen@kit edu

${ }^{2}$ Karlsruher Institut für Technologie (KIT), Institute for Biological Interfaces 1 - IBG-1, Hermann-vonHelmholtz-Platz 1, 76344 Eggenstein-Leopoldshafen, Germany, tim.scharnweber@kit.edu

${ }^{3}$ University College London, Department of Chemical Engineering, London WC 1E 7JE, United Kingdom. Now at Geochemistry Department, Sandia National Laboratories, Albuquerque, New Mexico 87185, USA, taho@sandia.gov

${ }^{4}$ University College London, Department of Chemical Engineering, Roberts Building, West Wing, Room 209A, Torrington Place, London WC 1E 7JE, United Kingdom, a.striolo@ucl.ac.uk

${ }^{5}$ Physics Department, Johannes Gutenberg University, Staudingerweg 7, 55128 Mainz, Germany, sulpizi@uni-mainz.de

${ }^{6}$ Karlsruher Institut für Technologie (KIT), Institute of Meteorology and Climate Research - IMK, Hermann-von-Helmholtz-Platz 1, 76344 Eggenstein-Leopoldshafen, Germany,

ahmed.abdelmonem@kit.edu

* Corresponding author:

Ahmed Abdelmonem

Karlsruhe Institute of Technology (KIT)

Institute of Meteorology and Climate Research - Atmospheric Aerosol Research Division

Hermann-von-Helmholtz-Platz 1, 76344 Eggenstein-Leopoldshafen, Germany

Tel : $+49(0) 72160824520$

Mobile : $+49(0) 1799799584$

FAX : :+49 (0) 72160824332

E-Mail: ahmed.abdelmonem@kit.edu 


\begin{abstract}
We present a measurement cell that allows simultaneous measurement of second harmonic generation (SHG) and streaming potential (SP) at mineral-water interfaces for flat specimen that are suitable for non-linear optical (NLO) studies. The set-up directly yields SHG data for the interface of interest and can also be used to obtain information concerning the influence of flow on NLO signals from that interface. The streaming potential is at present measured against a reference substrate (PTFE). The properties of this inert reference can be independently determined for the same conditions. With the new cell, for the first time the SHG signal and the SP for flat surfaces have been simultaneously measured on the same surface. This can in turn be used to unambiguously relate the two observations for identical solution composition. The SHG part of the cell with a fluorite sample confirmed previously observed differences in NLO signal under flow vs. no flow conditions in sum frequency generation (SFG) investigations. . As a second test surface, an inert ("hydrophobic") OTS covered sapphire-c electrolyte interface was studied to verify the zeta-potential measurements with the new cell. For this system we also obtained combined zeta-potential/SHG data in the vicinity of the point of zero charge. The zeta-potential was found to be proportional to the NLO signals. Furthermore, on the accessible time scales of the SHG measurements no effects of flow, flow velocity and stopped flow occurred on the interfacial water structure. This insensitivity to flow for the inert surface was corroborated by concomitant molecular dynamics simulations. Finally, the set-up was used for simultaneous measurements of the two properties as a function of time in automated titrations with an oxidic surface. Surprisingly different polarization combinations obtained in two separate titrations, yielded clearly different SHG data, while under identical conditions zetapotentials were exactly reproduced. One polarization combination scaled with the zeta-potentials, while the other did not. The work provides an advanced approach for investigating liquid/surface interactions which play a major role in our environment. The set-up can be upgraded for SFG studies. which will allow more detailed studies on the water structure at a given interface, but also the combined study of specific adsorption including kinetics in combination with electrokinetics.
\end{abstract}

Keywords: zeta-potential, second harmonic generation, hydrophobic surface, molecular dynamics, solid-liquid interface 


\section{Introduction}

Solid-liquid interfaces play a major role in many contexts from industry to aquatic and atmospheric environmental topics. At most interfaces protons and hydroxide ions are involved in the interfacial charging, frequently as potential/charge determining components.

Surface charge and potential due to these ions are crucial in understanding and modelling environmental and industrial processes. The "inner" surface potential is maybe the most important but least accessible propertiy of the electrical double layer and the origin of it. As a consequence its determination via spectroscopic methods some 25 years ago [1] continues to be of interest. Recent examples address for example how surface potential affects the ice nucleation properties of atmospheric minerals [2-5], which cannot be addressed by classical solid solution approaches. Charging at classical solid solution interfaces has been traditionally studied by electrokinetics and potentiometric methods [6-8]. Less frequently applied approaches involve non-linear optical (NLO) methods [9-11] that are non-invasive and can cover salt concentrations beyond those accesible to the classical methods [12]. The latter are appropriate either at the higher salt levels (potentiometric titrations, more salt means increased proton interaction) or lower salt concentrations (electrokinetics, less salt means reduced screening). A current discussion exists on the meaning of NLO signals from solid-liquid interfaces concerning amongst other issues the extent of various electrical double layer contributions [12-15]. The pioneering work of Ong et al. on fused silica/aqueous electrolyte interfaces [1] and related second harmonic generation (SHG) data to "surface potential". The SHG signal depends on the second order nonlinear susceptibility, $\chi^{(2)}$, of the interfacial molecules that are asymmetrically orientated at the interface, and the third order nonlinear susceptibility, $\chi^{(3)}$, of the bulk water molecules oriented due to the static electric field of a charged surface $[1,12,16]$. Attempts to quantify are available $[12,14,15]$, but in particular the $\chi^{(3)}$ contribution, which is related to the static electric field at the interface, will strongly depend on what kind of surface potential is involved. Concering the salt dependence of the NLO signal, the strong effect of salt and the ability of a Gouy-Chapman approach to model it would rather point to the electrokinietic/DLVO potential (the diffuse layer potential) than to the "inner" surface potential. A more detailed discussion associated with typical experimental data from the literature can be found in Supplementary Information.

In some of the available models (single plane models) only one potential occurs, which then automatically is the surface potential. In multi-layer models, different distinct potentials occur and these have to be clearly distinguished. The most advanced ones attempt to cover the complete double layer sometimes from a truly mechanistic point of view [17]. Besides the question which interfacial potential should be used to interpret NLO experiments (i.e. surface potential or diffuse layer potential?), the comparison of a set of data on single crystal surfaces suggests that this might for example depend on how the experiment is carried out. SHG data on sapphire-c (the basal, i.e. (0001), plane of $\alpha-\mathrm{Al}_{2} \mathrm{O}_{3}$ ) obtained through the liquid by Fitts et al. [18] produced a point of zero charge, which is close to independently measured isoelectric points [19-21]. Thus the data by Fitts et al. would mimic the zeta-potential vs. pH curves obtained by others [20], whereas SHG measurements through the crystal [21] instead produced data that are clearly reminiscent of the expected "inner" surface potential vs pH curve [20, 22], see SI. Unfortunately, such seemingly contradictory results can be conveniently explained by differences in sample origin or preparation [21]. Thus ambiguity will remain if one attempts to compare data from different laboratories. The 
solution to this is to measure the data of interest simultaneously on one sample to avoid this ambiguity.

While previous modeling work reporting NLO data has been involving purely diffuse layer models mainly (sometimes in exchange with a constant capacitance model, i.e. another single plane model, at higher salt levels), recent work [23] has used, in the case of a charged water interface, an electrical double layer model that involves separation of the NLO signal contribution originating from the "bonded interface layer (BIL)" from contribution due to the diffuse layer. The former was attributed to the $\chi^{(2)}$ contribution to the total resonant susceptibility and might be associated with the Stern layer, while the latter is associated to the diffuse layer and is proportional to the dc electric field. . The $x^{(3)}$ contribution is related to the electric field and most of the available attempts to quantification resort to a Gouy-Chapman type equation. This usually concerns data at constant $\mathrm{pH}$, with variation of salt level, and although exceptions exist $[24,25]$, most modelling studies involve erroneous assumptions, namely that proton related surface charge density does not change with the concentration of background electrolyte concentration [14] or the concentration of specifically adsorbing ions [25].

The dependence of NLO signals on salt level is surprisingly well described by Gouy-Chapman type approaches, while it is known from classical work on oxide interfaces that this approach fails to correctly describe the protonation/deprotonation behaviour even at low salt levels. As a consequence the purely diffuse layer approach can hardly yield accurate values for the "inner" surface potential. From this point of view NLO signal should rather scale with DLVO (diffuse layer) potentials as obtained from Stern-Gouy-Chapman models discussed above.

To come to a more profound understanding of for example the relation between the NLO and the classical (electrokinetic) data simultaneous measurements of electrical double layer properties and NLO signals under identical conditions and for the same sample are required. The aim of the present work was therefore to design a cell capable of measuring SHG and streaming potential (SP) for flat surfaces. Although flat surfaces may have the advantage of surface structural control, it has been argued that the interfacial behavior of flat oxide mineral surfaces may be affected by secondary phenomena like preferential physical adsorption of hydroxide ions at $\mathrm{pH}>4[20,26]$. We suggest that with a combined set-up to measure both a macroscopic interfacial potential and the molecular structure based NLO signal a more rigorous and less ambiguous relation between quantities can be obtained. At the same time such measurements avoid the sample preparation/origin problem and will ultimately help to relate NLO signals to the corresponding potentials in the electrical double layer. Alternatively such set-ups will show that the relation is not trivial.

As a step into this direction we designed a cell that allows to measure the zeta-potential and the SHG signal on one surface. The set-up was tested in scoping applications on three different systems.

First we use the newly designed cell to verify the SHG capabilities by testing the fluorite-water interface for flow and no-flow conditions, under equilibrium and non-equilibrium conditions. This part was intended to verify the NLO signal under flow against existing data [27]. No attempt was made to extract zeta-potentials for the non-equilibrium conditions.

As a second system we chose an inert surface to study the relationship between NLO signal and zetapotential close to the IEP and to minimize non-equilibrium effects. Also at present, the streaming potential in the combined flow cell is measured against an inert (PTFE) surface. Since inert surfaces 
exhibit generic behavior with IEP pH 4, an OTS (octadecyltrichlorosilane)-coated sapphire-c is an ideal test system because its interface with water is accessible to NLO, and it is expected to show pHdependent charging very similar to PTFE. With this system we studied the extent to which flow would affect interfacial water structure, compared to the same system under no-flow conditions.

The third system tested was an aged sapphire-c surface with an intermediate IEP. It exhibits traditional zeta-potential vs. $\mathrm{pH}$ behavior of an oxide surface and should for example avoid the hydroxide adsorption problem that is still under debate for inert surfaces. In the experiments we obtained for the first time a direct comparison between the zeta-potential and NLO signal for identical samples and under identical conditions over a wide range of $\mathrm{pH}$-values.

We stress that the cell can be used for the combined measurement with any flat interface that is appropriate for NLO studies under the geometrical constraints of the cell. Ultimately, knowing both zeta-potential (as an approximate value for the diffuse layer potential) and "inner" surface potential might allow to quantify the NLO signal from well constrained interfacial properties, ideally without the need to involve surface complexation models. The present experimental development is a first step towards this goal.

\section{Materials and Methods}

\section{Materials}

Ultrapure water $(18.2 \mathrm{M} \Omega \mathrm{cm})$ with total organic content below $4 \mathrm{ppb}$ was used to prepare all solutions. The $\mathrm{KCl}$ salt (suprapur, Merck) was dried in an oven at $120{ }^{\circ} \mathrm{C}$ prior to use. For $\mathrm{pH}$ adjustment we used $\mathrm{KOH}$ ( $50 \mathrm{mM}$, Kraft) and $\mathrm{HCl}(50 \mathrm{mM}$, prepared from $1 \mathrm{M}$ standard, VWR).

Three distinct sample surfaces were used, i.e.

(i) a fluorite prism (equilateral, i.e. $60^{\circ}, 25 \mathrm{~mm} \times 25 \mathrm{~mm} \times 25 \mathrm{~mm}$ from Crystran, Dorset, UK) in contact with $\mathrm{NaF}$ (p.a., Riedel de Haen) or $\mathrm{HCl}$ of known concentration (prepared from $1 \mathrm{M} \mathrm{VWR}$ standard) solutions,

(ii) a sapphire-c prism (Kyburz, Safnern, Switzerland) and sapphire-c single crystals (Mateck, Aachen, Germany) that were coated by OTS, and

(iii) a sapphire-c prism (Kyburz, Safnern, Switzerland) that had been aged for several monts in MilliQ water until it produced an IEP distinctly above the IEP of 4 obtained for unaged sample of the same sources.

The sapphire-c prism that was aged had the geometry of the fluorite prism. The sapphire-prism that was coated with OTS was equilateral as well, but had dimensions of $15 \mathrm{~mm} \times 15 \mathrm{~mm} \times 15 \mathrm{~mm}$.

\section{Surface preparation}

To produce the inert surface, i.e. the OTS-coated sapphire-c water interface, a prism sample was used that exposed the c-plane as the hypotenuse. This sample was used for combined SHG/SP measurements. Flat window sapphire-c crystals (Mateck, Aachen, Germany) were coated in the same 
way for contact angle-measurements. All sapphire-c substrates were covered by OTS using previously published methods [28]. The resulting contact angle (sessile drop) was around 70 degrees, which is somewhat lower than the 90 degrees contact angle obtained elsewhere [28]. The chemicals used were octadecyltrichlorosilane (OTS, p.a., Merck), toluene (p.a., Merck), sulfuric acid 95-97 \% (p.a., Merck), and hydrogen peroxide $30 \%$ (p.a., Merck).

The measurements with the fluorite sample were done after the experiments with the OTS-covered surface, because the data with the OTS system did not reveal any effect of flow. To verify the set-up with a system that is known to produce flow effects, the fluorite prism from the same source as previously used for the SFG study by Lis et al. [27] was tested. The sample was prepared as described by Lis et al. [27].

The series of measurements with an uncoated sapphire-c prism involved a specimen that had been extensively used in previous measurements with simple $\mathrm{KCl}$ and $\mathrm{NaCl}$ electrolytes and was subsequently kept for several months in MilliQ water. . Prior to use in the present work, this aged sapphire-c sample was cleaned according to previously described procedures [20, 29]. Measurements with the unaged prism revealed the behavior as a function of $\mathrm{pH}$ that is expected for an ideal sapphire-c surface with IEP around $\mathrm{pH} 4$ [21]. Preliminary measurements with the aged sample showed that the IEP was above $\mathrm{pH}$ 5.5. Modifying the surface of fresh sapphire-c by adding dissolved Al changes the behavior and increases the IEP up to $\mathrm{pH} 9$ [30]. Although this can be partially reversed, the IEP stabilizes above $\mathrm{pH} 5$ according to previous studies [30] as does the aged sample.

\section{Set-up and cell description}

\section{Second Harmonic Generation component}

SHG is a powerful and simple surface-sensitive spectroscopic tool for studying interfacial molecules $[9,31]$. The amplitude and polarization of the generated field, as a function of the polarization of the incident fields, carry information on the abundance and structure of the interfacial molecules between two isotropic media [32-34]. Our SHG set-up has been previously described in some detail [35]. In brief, two femtosecond laser pulses at $800 \mathrm{~nm}$ are mixed at a solid/water interface in a copropagating, total internal reflection (TIR) geometry from the prism side to generate the SHG light at the prism hypotenuse/water interface. The TIR geometry provides additional enhancement of the SHG signal if the incident beams are close to the critical angle, as in this work. While some experiments were carried out with a single fundamental beam, in other experiments, two incident beams at two slightly different angles were applied. The two beams were allowed to overlap in space and time at the interface, mimicking SFG set-ups. This allows better separation between the background accompanying the fundamental beam and the signal from the probed interface. In the single incident beam experiments, the PP (P-polarized SHG output, and P-polarized beam 1 input) polarization combination was used. In the experiments with two incident beams, PPP (P-polarized SHG output, P-polarized beam 1 input, and P-polarized beam 2 input) and SSP (S-polarized SHG output, S-polarized beam 1 input, and P-polarized beam 2 input) polarization combinations were used. The advantage of the SSP configuration is that only one component of the surface nonlinear susceptibility tensor, $\chi_{y y z}^{(2)}$, is probed independently of the incident angle. The PPP (or PP) configuration, instead, probes four components $\left(\chi_{x x z}^{(2)}, \chi_{x z x}^{(2)}, \chi_{z x x}^{(2)}\right.$ and $\left.\chi_{z z z}^{(2)}\right)$ with respective weights 
depending on the angle of incidence [34, 36]. Only for the incident angle equal to the critical angle of TIR all components are zeroed except $\left(\chi_{z z Z}^{(2)}\right)$. In all of the SHG experiments, the signal was collected at the liquid/sample interface using TIR geometry under either no-flow or flow conditions

\section{Streaming Potential component}

In the streaming potential part of the experiments a solution is forced through a flow channel of well-defined geometry under a given applied pressure. The commercial SurPass apparatus (Anton Paar, Graz, Austria) for streaming potential measurements was adapted for use with the new cell. Specifically the electrodes and pressure sensors were used outside of the apparatus and connected to the new cell mounted to an optical table in such a way that the SHG could be measured on a given sample prism / water interface. The newly designed flow cell (Fig. 1) was rebuilt in PTFE from the inset of the standard clamping cell of the SurPass. As a consequence, on of the two relevant surfaces in the flow channel is a PTFE surface. The standard SurPass seal films serve as spacers to generate a defined channel geometry, see Fig. 2 . In cases where the prism was sufficiently large (fluorite and aged sapphire prisms) the standard channel can be used. For prims with too small dimensions a separate PTFE block can be designed to adjust the flow channel accordingly. The cylindrical electrodes and the pressure sensors of the standard SurPass equipment can also be used. The SurPass software collects all the data and stores them. The zeta-potential of the SHG probed surface is measured with respect to a reference surface (i.e. the PTFE side of the channel). The PTFE properties are determined independently with the same cell geometry, which allows the zetapotential of the sample surface that is probed by SHG to be calculated from the equation

$\zeta_{\text {sample }}=2 \zeta_{\text {exp }}-\zeta_{\text {ref }}$

In this equation $\zeta_{\text {sample }}$ is the zeta-potential of the sample surface of interest, i.e. here the one that is examined via NLO, $\zeta_{\text {exp }}$ is the experimentally observed zeta-potential, caused by flow of the solution through the channel bounded by the two surfaces, and $\zeta_{\text {ref }}$ is the known zeta-potential of the reference surface (here PTFE).

To ensure that a change in channel dimension does not affect the measurements, the reference measurements were performed with identical flow channels. For the OTS system the measurements showed that sample of interest and reference sample were behaving identically. Furthermore, the reference surface with the reduced channel gave the same results previously reported for PTFE [37].

\section{Combined measurement set-up}

The PTFE block is fixed to the optical table by a screw (Figure 1) in such a way that it will not move during the measurements involving flow of solutions. The prisms used for measurement can be pressed against the spacer that generates the flow channel. The internal SurPass pumps can be used (for example in rinse mode to give constant pressure or in a typical streaming potential experiment to generate pressure ramps). Alternatively, an external pump can be used to generate constant pressure for extended periods and increase the applied pressure above the SurPass limits. No leakage was observed during our experiments. Only when the pressure was increased above 1000 mbar using the external pump, we observed leakage. As a consequence attempts to generate turbulent flow were discontinued for the time being. The SurPass $\mathrm{pH}$ and conductivity electrodes are 
used in the solution reservoir. The solution can be pumped in a closed circle or involve fresh solution that is pumped through the cell towards a waste.

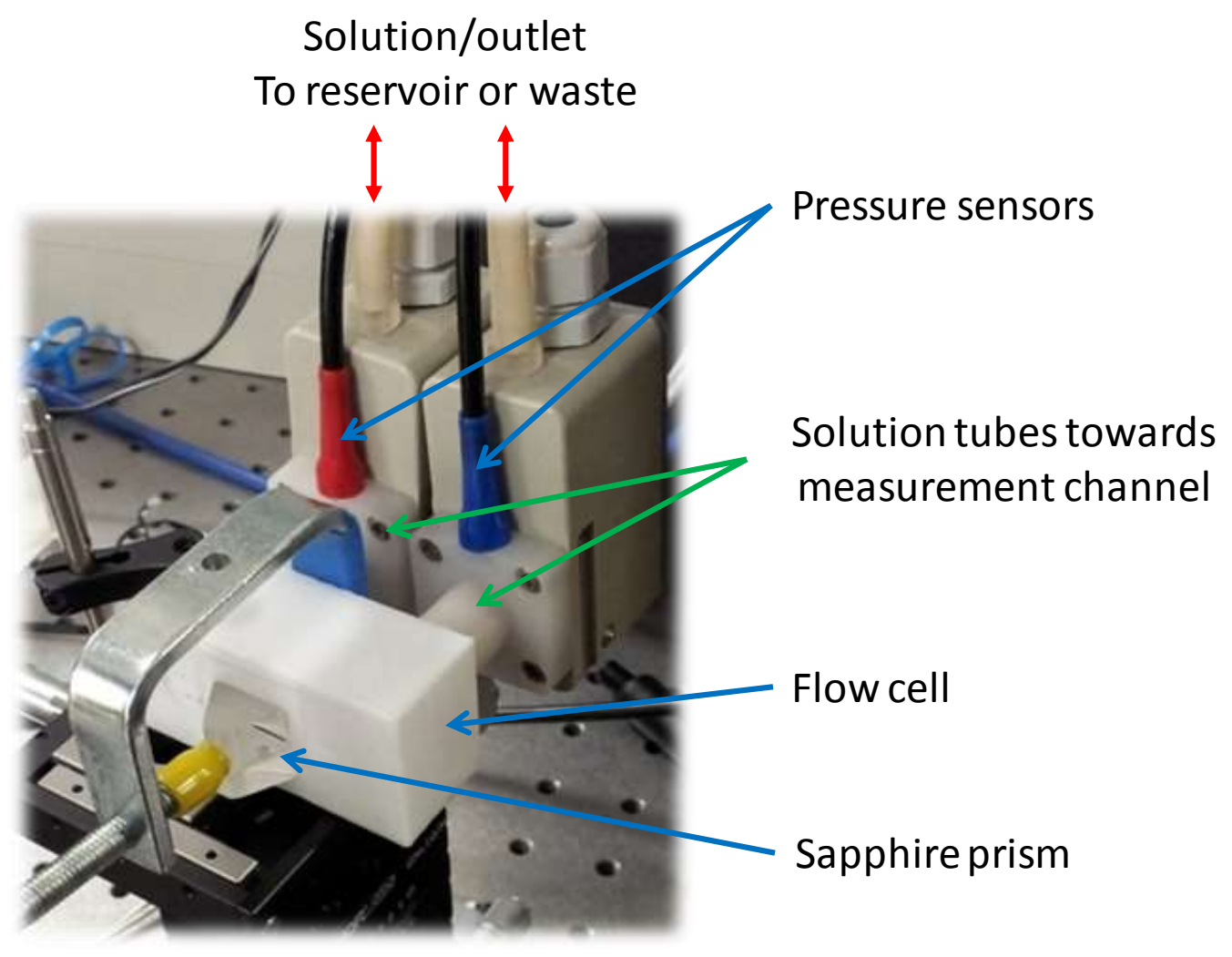

Figure 1. Sample cell used for simultaneous SHG and streaming potential measurements. The cell is made of PTFE. The flow channel is about $150 \mu \mathrm{m}$ thick, $25 \mathrm{~mm}$ long and $10 \mathrm{~mm}$ wide in the case of the sample prism used here (Sapphire prism). Pressure sensors and measurement electrodes are those supplied with the Anton Paar apparatus. Solution can be supplied from reservoir either via the SurPass pumps, or via an external pump. Solutions can be pumped in a closed circle, or from a reservoir to a waste container. The electrode and pressure measurements, as well as data recordings are controlled by the Anton Paar software. 


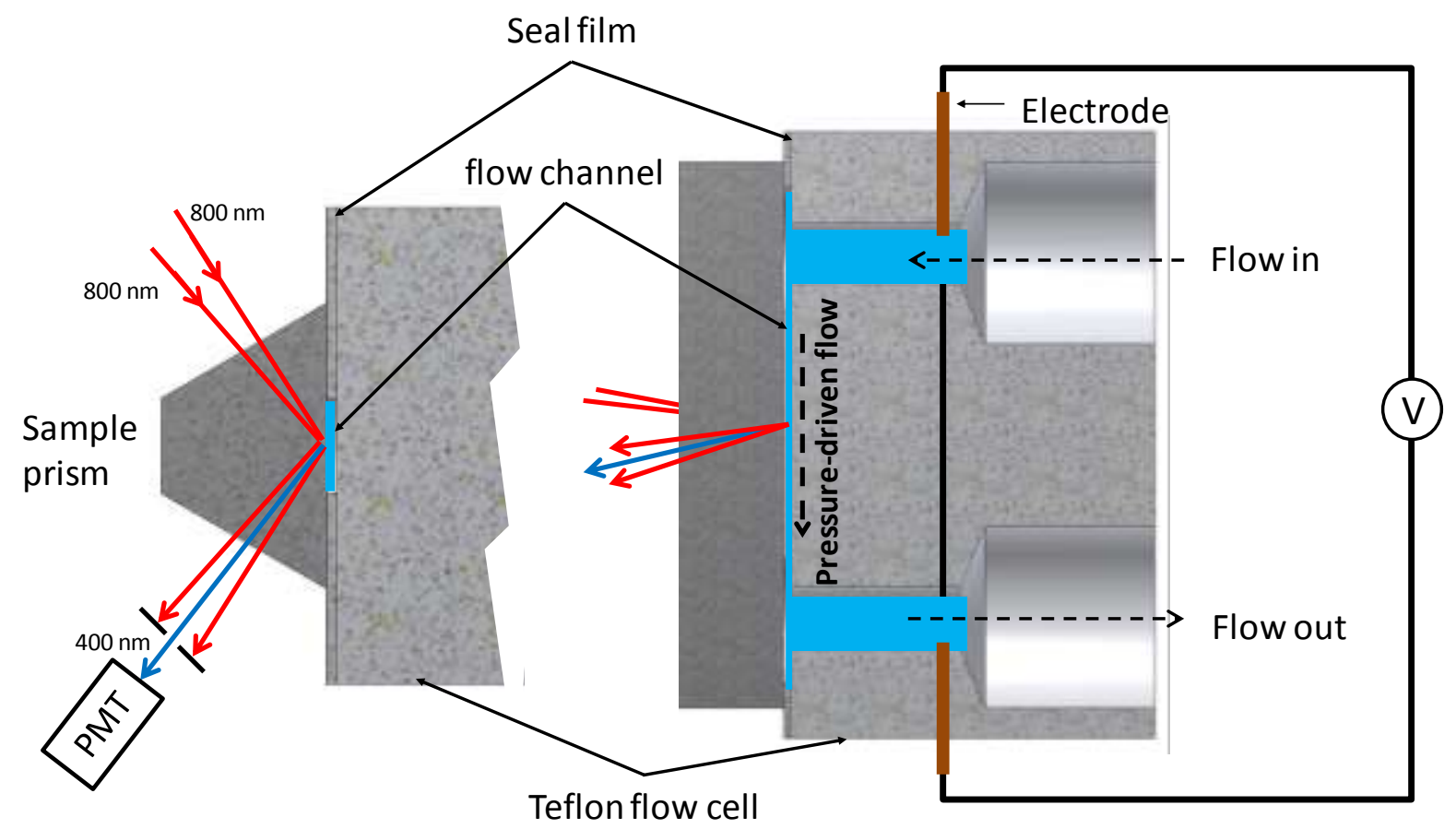

Top cross-section

Side cross-section

Figure 2. Schematic drawing illustrating the design of the flow cell used to measure SHG and streaming potential simultaneously. The left panel shows the cell turned by 90 degrees. The streaming potential was measured under pressurized flow while the SHG signal was collected at the liquid/sample interface using TIR geometry.

The experiments were carried out in a thermostated room at $20.5^{\circ} \mathrm{C} \pm 0.1{ }^{\circ} \mathrm{C}$. All solutions and samples were stored in the room for a sufficiently long period to assure constant temperature conditions.

\section{Results and discussion}

\section{SHG on the fluorite system}

Figure 3 shows the SHG results obtained on the fluorite prism with non-equilibrium (Figure 3a) and equilibrium solutions (Figure 3b). Our experiments replicate conditions by Lis et al. [27]. The NLO signal was recorded in PPP configuration and flow was generated with an external pump. The results show that flow of non-equilibrium solutions strongly affects the NLO signal from the fluorite aqueous solution interface, in agreement with what has been previously observed by Lis et al. [27] by SFG. Flow enhances the signal, and stopping the flow caused a gradual relaxation of the SHG signal towards the no-flow value as previously observed by Lis et al. [27]. Increasing the pressure resulted in a stronger SHG signal. . The black squares at the no-flow points indicate conditions prior to switching the flow on. The higher the applied pressure, the longer it takes for the signals to relax once the flow is stopped. For the two highest applied pressures no attempt was made to retrieve the no-flow signal. Furthermore, higher flow velocities appear to cause much stronger fluctuations in the 
signal. The SHG results support the hypothesis that the fluorite surface is strongly perturbed by continuous flow of a non-equilibrium solution. Such NLO data can conveniently be used to assess whether a system is at equilibrium or at steady state under given conditions. Our results clearly show that with non-equilibrium solutions a fluorite surface is not even at steady state for extended durations of applied constant pressure. For a reasonable streaming potential experiment however the variation of the equilibrium state of the surface with applied pressure needs to be excluded. To verify if a steady state can be obtained, longer measurement times were applied at 200 mbar applied pressure. The variation of the SHG signal indicates changes in surface chemistry even after 1000 seconds. Non-equilibrium states such as those shown in Figure 3a would also occur during a pressure ramp (where in typical experiments the applied pressure goes from 100 mbar to 300 mbar within 30 seconds). However, the SurPass software would not allow to identify such a non-equilibrium state. Therefore, it is attempted in the usual experimental procedure to avoid the occurrence of nonequilibrium situations during the pressure ramps via equilibration of the surface with the respective solution in a rinse cycle (closed system). The duration of this equilibration step prior to the pressure ramps is user-defined.

The strong flow effect initially reported by Lis et al. [27] was interpreted in the sense that the fresh solution that is constantly supplied leads to continuous changes at the surface. The NLO signal suggests that water molecules are strongly affected during these changes. With the common interpretation of the NLO signal in terms of surface potential, the data would suggest that much stronger electric fields occur under the non-equilibrium conditions i.e. during dissolution, compared to equilibrium or no-flow conditions. For no-flow conditions local equilibrium is probably quickly established and then remains unperturbed. More detailed discussions on this can be found in the original paper, but we believe that more experimental work, for example with defined undersaturation in the solutions, could yield valuable new insight in dissolution of mineral surfaces.

Based on the results with the fluorite crystal, one obvious advantage of our cell is that we can easily verify if a given surface is in equilibrium (or exhibits a steady state) in contact with a given solution. Equilibration may be a problem for rather soluble solids such as calcite or fluorite. In principle the solution compositions for such systems at equilibrium are controlled by the solubility. Equilibration during the SurPass measurements would take a long time for the inherent high solution volumes (500 $\mathrm{ml}$ ) and small surfaces ( $4 \mathrm{~cm}^{2}$ in the gap cell). One simple way to avoid this problem is to use solutions that inhibit dissolution. As shown in Figure $3 \mathrm{~b}$ the presence of $4 \mathrm{mM} \mathrm{NaF}$ (which suppresses dissolution) eliminates the flow effect. The zeta-potential measured for the conditions of Figure $3 \mathrm{~b}$ was $(11.8 \pm 1.6) \mathrm{mV}$. 
a)

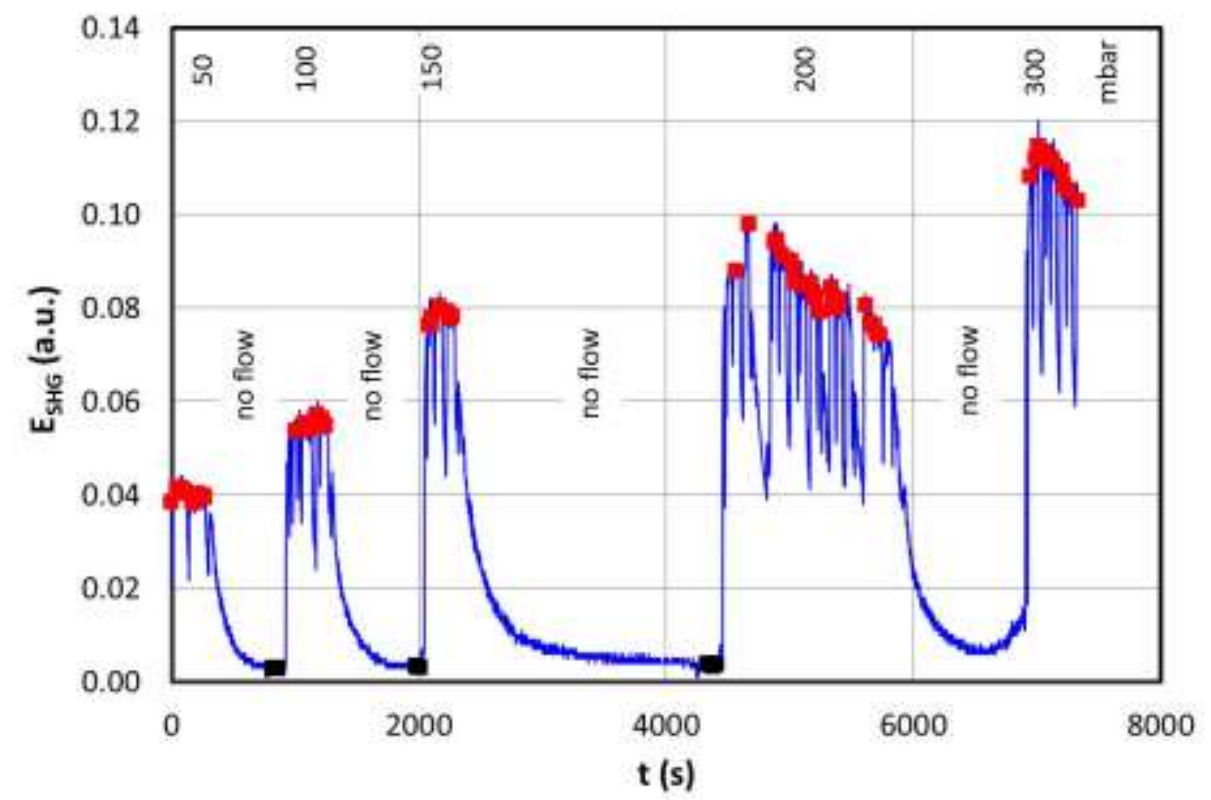

b)

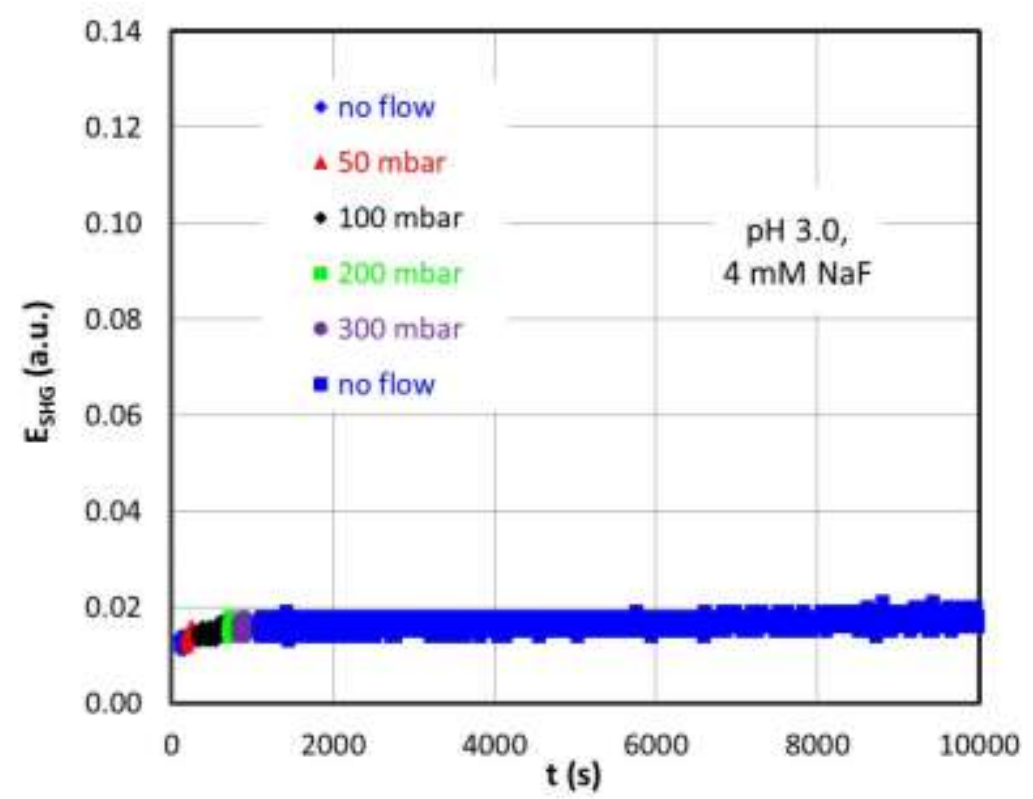

Figure 3. Effect of flow (as given by the applied pressure in mbar) on the SHG signal from a fluorite surface at (a) pH $2.6(\mathrm{HCl})$ and $(\mathrm{b})$ in the presence of $4 \mathrm{mM} \mathrm{NaF}$ at pH $3(\mathrm{HCl})$. The scales of the $\mathrm{y}$-axes are the same to facilitate direct comparison. 


\section{Separate SHG and SP measurements on OTS coated sapphire-c}

As a second sample an OTS coated sapphire-c prism, exposing an inert surface to the NLO measurements, was considered because (i) flow effects in the SHG signal due to substrate dissolution were not expected, and (ii) the PTFE reference used in the zeta-potential measurement is another inert surface. Flow or aging effects would have occurred if the coating had been deteriorating during the period of the measurements, but this was not observed.

Figure 4a shows SHG results for the OTS-sapphire-c/aqueous solution interface. The experiments were conducted in a similar way as for the fluorite surface. Only one beam was used in this measurement series, which facilitates alignment. On Figure 4a, we plot the SHG field for PP configuration as a function of the applied pressure for three different $\mathrm{pH}$ values. No effect of flow is observed based on the absence of a change in the SHG signal for flow and no-flow conditions while changing the $\mathrm{pH}$ clearly affects the signal. The absence of a flow effect with time suggests that the interfacial water structure is not changing due to the flow. This is perhaps expected since on the inert surface we expect a slip-boundary condition.

Unlike for the fluorite system, the constant value of the SHG signal for a given $\mathrm{pH}$ and variable pressures indicates equilibrium. With this knowledge the Anton Paar device can be used to extract the potential difference $(U)$ between the two electrodes (Figure 1 ) at a given applied pressure. In Figure $4 \mathrm{~b}$ we compare data for constant pressure conditions with a standard pressure ramp (open symbols) obtained for the same conditions. The agreement obtained with different procedures proves that the use of the cell outside the SurPass apparatus and beyond the typical pressure ramps yields reliable data even when an external pump is used. This is important because with an improved cell, and with the use of an external pump we expect to be able to cover pressures beyond the range allowed by the Anton Paar device, which would eventually allow us to study turbulent systems. For turbulent systems we would expect changes in the water structure, when switching from no-flow to flow conditions. Ultimately, the set-up could then be used to study the onset of turbulence.

The IEP of the OTS surface sample was found at $\mathrm{pH} \approx 4$, which is typical for many inert materials [3840]. The IEP of the reference system (two PTFE surfaces) and the zeta-potential coincided with those of the system exposing the PTFE and the OTS-covered sapphire-c surfaces (i.e. when the OTS coated sapphire replaced one PTFE surface) so that corrections via equation (1) were not required. Figure 5 shows the relation between the determined zeta-potentials and the SHG signals for the three $\mathrm{pH}$ values studied. In Figure $5 a$ we use the constant pH SHG data (symbols) from Figure 4a, while in Figure $5 b$ the SHG are those obtained for flow conditions (lines in Figure $4 a$ ). Both sets of data suggest a linear relationship, but this is not surprising, since in the vicinity of the IEP, zeta-potentials are usually linear (see SI). Previous studies using SHG also report linear relationships between the second harmonic field and the $\mathrm{pH}$ [18], see SI. It is also remarkable that at the IEP, despite the reversal in the sign of net charge in the zeta-potential, no inversion of some kind occurs in the SHG signal, while it does in SFG data. The absence of such an inversion in SHG studies has been previously reported for numerous cases $[18,41]$ and requires the assignment of the point of zero charge via a point of zero salt effect. 
a)

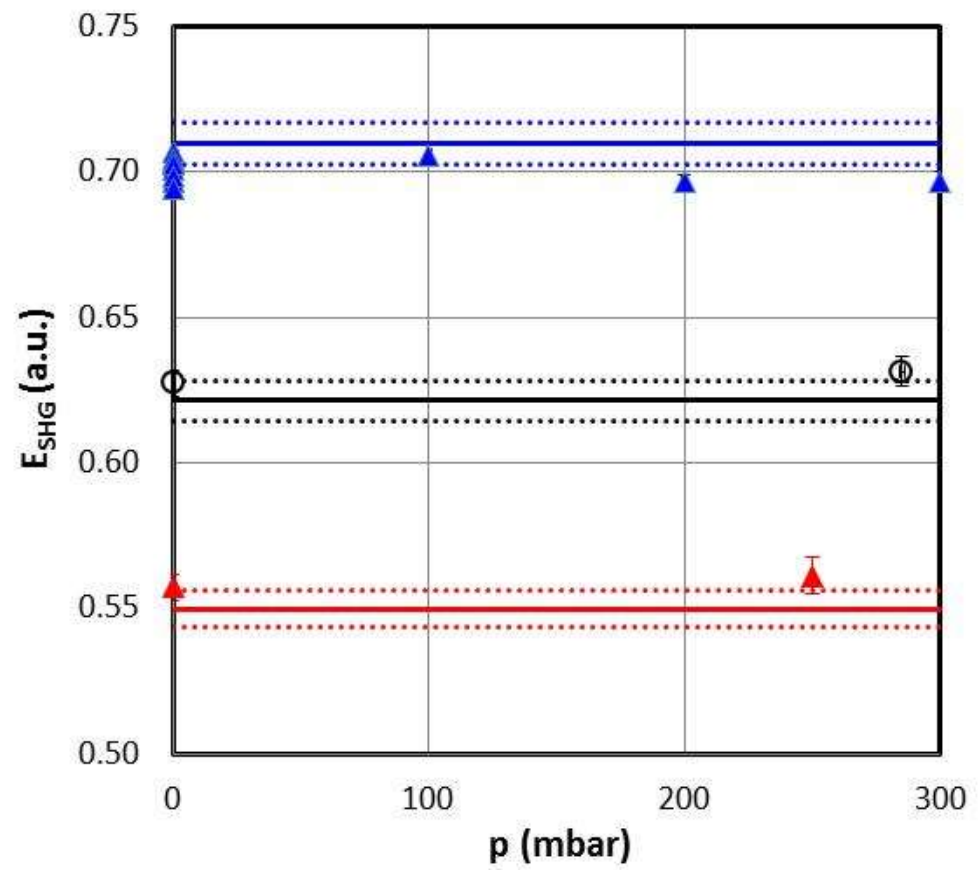

b) 


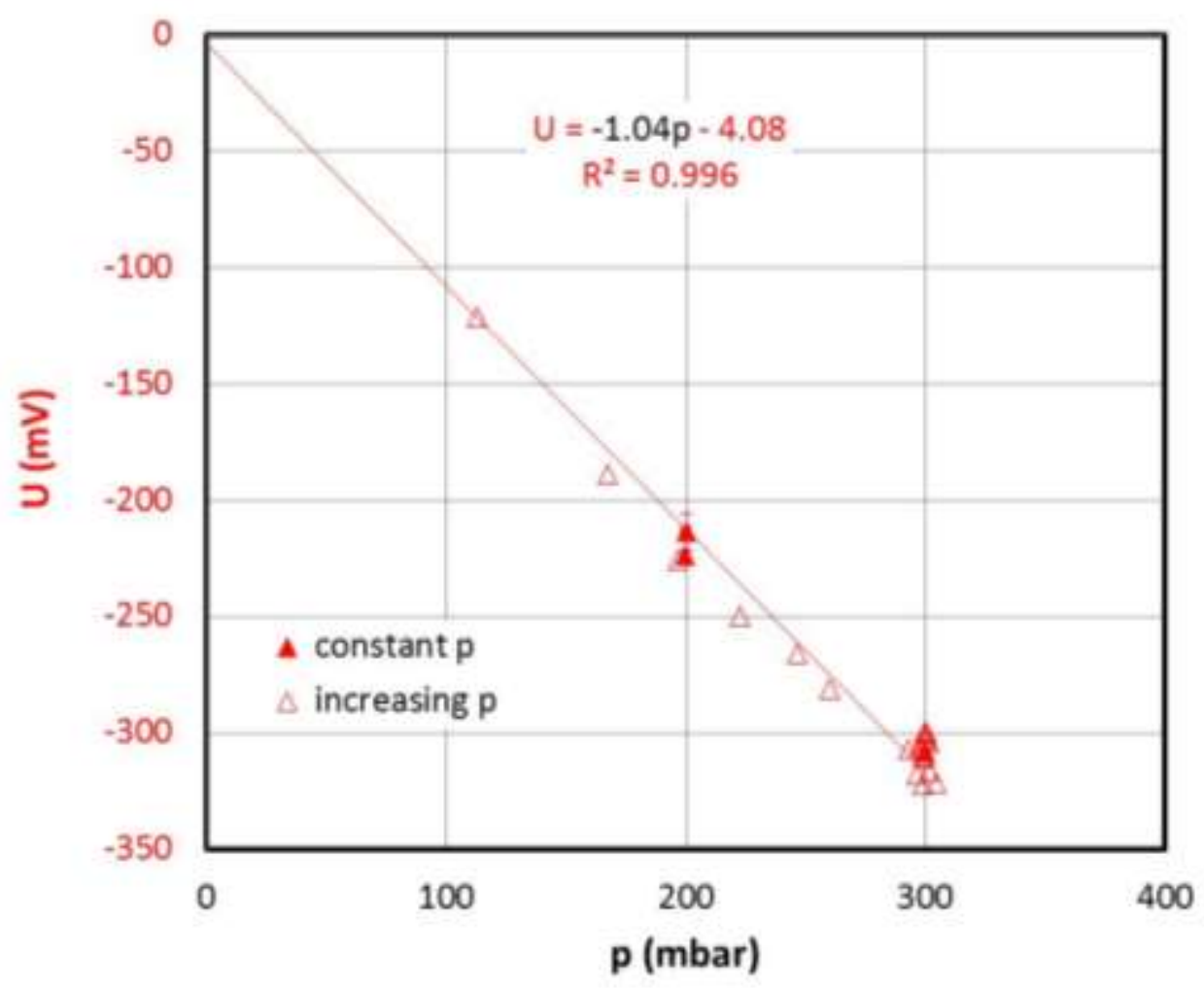

Figure 4. SHG and SP data for OTS-coated sapphire-c. a) SHG Field as a function of applied pressure for different $\mathrm{pH}$ values, no extra background electrolyte was added. The data points show results collected for constant pressure conditions at a given $\mathrm{pH}$. Error bars are standard deviation. For each $\mathrm{pH}$ value the full line corresponds to the SHG signal collected during the zeta-potential measurement. The two respective dotted lines for each $\mathrm{pH}$ are the error bars for these SHG data (standard deviations). b) Pressure ramp constructed from the measured potential (U) at constant pressure(p, full symbols, noted "constant $p$ ", the error bars correspond to standard deviations) as obtained during the series of SHG measurements at $\mathrm{pH} 4.6$ in a) and pressure ramp from a distinct streaming potential measurement with software control (open symbols, noted "increasing $\mathrm{p}$ ", here individual points are shown).

The observed linear relation between zeta potential and SHG signal does not imply that the SHG signal is caused by the interfacial potential obtained by the streaming potential technique, since the "inner" surface potential typically has a linear dependence on $\mathrm{pH}$ as well for most oxide minerals, as for example measured by Bousse and Bergveld for alumina between $\mathrm{pH} 2$ and 5 in $\mathrm{NaCl}$ solution [42] (see $\mathrm{SI}$ ) or at inert surface as measured for the gas water interface between $\mathrm{pH} 2$ and 3 in $\mathrm{HCl}$ solution by Kallay et al. [43]. Additional examples for the "inner" surface potential from the literature are discussed in SI. A clear difference between diffuse layer and "inner" potentials occurs under conditions sufficiently far from the point of zero charge, where the zeta-potential levels off, while the "inner" surface potential continues to have a linear relationship with $\mathrm{pH}$. Experiments have been reported in which combined streaming potential and surface potential measurements were carried out $[44,45]$. These are shown in SI, and clearly show that zeta- and "inner" surface potential will cease to be proportional. In SI we also discuss salt dependence. From the available information, it might be concluded that the "inner" surface potentials of many minerals does not strongly depend 
on "inert" electrolyte concentration, while zeta-potentials or DLVO-potentials show the typical double layer screening behavior, which is found in SHG experiments as well (see SI).

a)

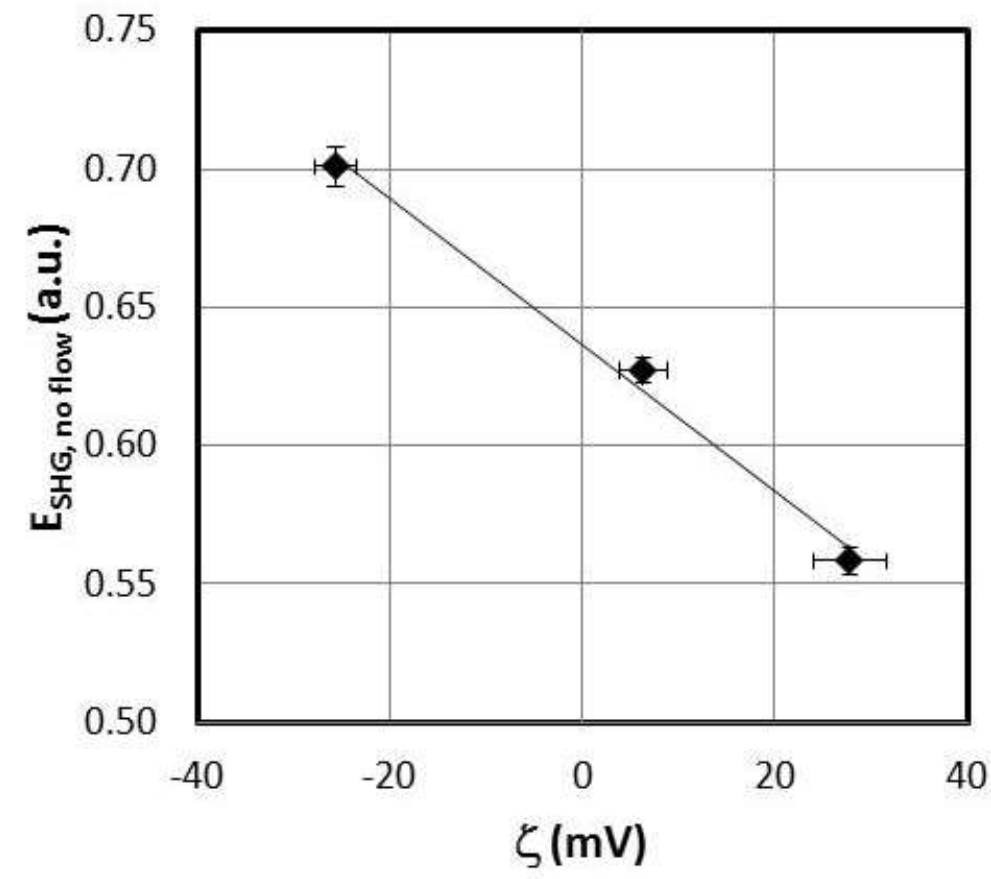

b)

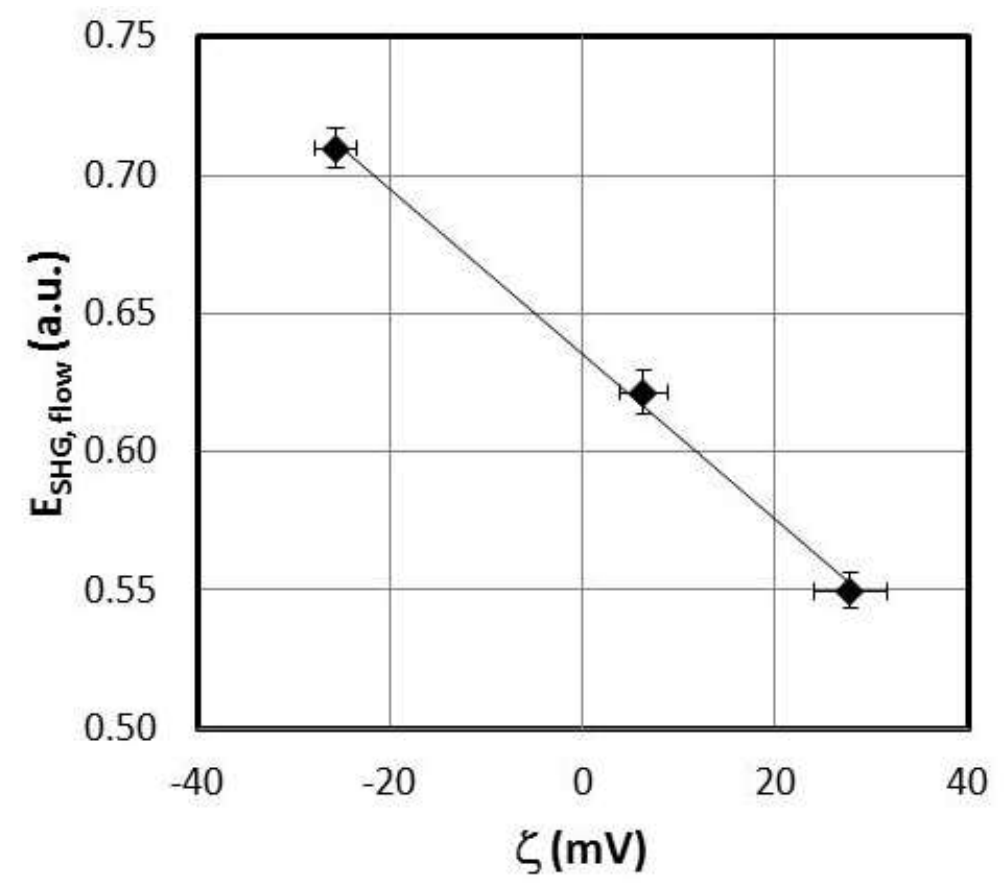

Figure 5. Relation between measured zeta-potential using conventional ramps and non-linear optical signal for OTS covered sapphire-c for no flow conditions (a) and flow conditions (b). The IEP is found close to $\mathrm{pH} 4$, as for most inert materials. Error bars are standard deviations. 
Since the data from Figures 4 and 5 conta far unpublished information, we will discuss in detail the absence of a flow effect on the interfacial water dipole orientation observed on a hydrophobic surface based on molecular dynamics (MD) simulations. While such absence can be taken as an indirect piece of evidence that the flow effects observed in systems with significant solubility (like fluorite [27], c.f. Figure 3a) are indeed related to dissolution, the expected absence of such dissolution reactions in the case of the OTS-covered sapphire crystal suggests that interfacial water structure is insensitive to flow or no-flow conditions. In other words, when switching on flow the interfacial water molecules are just pushed through the channel without re-orienting. To assess the validity of this hypothesis, we relate the experimental measurements to all atom molecular dynamics (MD) simulations for water on a hydrophobic substrate. Although the experimental and the simulated substrates are different, as clearly manifested, e.g., by the contact angle, which is lower in the experimental case than in the simulated system, the MD simulation results support the experimentally observed absence of a flow effect.

The simulations are conducted implementing protocols described elsewhere [46, 47]. More details are given in SI. In short, a model surface is prepared using a cubic crystal lattice and maintained rigid. To estimate the contact angle a small droplet of water is placed on the surface, and analyzed once equilibrium has been achieved. The procedure mimics the experimental sessile drop approach. To mimize computional cost, the drop was kept so small that the line tension is expected to affect the calculated contact angle. Although an alternative approach to simulate contact angles in simulations [48], for the current purpose our prior approaches were deemed sufficient since a the contact angle is not necessarily the most important descriptor for the observation of hydrodynamic slip at the liquid-solid interface [49]. The strength of interactions between the atoms in the solid and the water molecules, the distribution of the solid atoms on the surface, and the physical matching between the size of the water molecules and the lattice of the atoms in the solid are all important parameters. Our primary goal was to generate hydrodynamic slip. For the estimation nof the contact angle with our previous approaches a drop with 1,000 SPC/E water molecules was simulated [50]. Interactions between solid atoms and water molecules are described via dispersive interactions alone (i.e. via Lennard-Jones potentials) with parameters obtained from those of the pure compounds via LorentzBerthelot mixing rules. The Lennard-Jones parameters for the solid are tuned until the contact angle is above 90 degrees. To quantify the effect of flow on the properties of water molecules within the hydration layer, we set-up a $2 \mathrm{~nm}$ wide slit-shaped channel, filled it with water, and imposed flow while maintaining the solid substrate rigid. The algorithm is similar to that previously implemented [51]. In the flow simulations, the surfaces are kept rigid and water flow is in the direction parallel to the surface due to an imposed acceleration of $1 \mathrm{~nm} / \mathrm{ps}^{2}$ forced on each water molecule. Figure 6 shows the simulation snapshot for the system used to quantify the contact angle (top panel) and that for the system used to quantify the effect of flow (middle panel). The bottom panel of Figure 6 shows the velocity of water molecules as a function of their position within the slit-shaped pore once the flow has been imposedlt can be seen that the velocity profile is flat across the pore, indicating plug flow, so that hydrodynamic slip is achieved.

The major purpose of the simulations was to find out whether the imposed flow affects the structure of water molecules within the hydration layer that would be probed by SHG. The density profiles of oxygen and hydrogen atoms of water molecules within the slit shaped pore and the orientation of water molecules within the first hydration layer, identified by the density profiles, are shown in 16 
Figure 7 for no-flow and for fully established flow. No discernible difference is observed in the density profiles (top panels) or concerning the orientation of water molecules (bottom panels) whether flow occurs (right panels) or not (left panels). Regarding the orientation of the water molecules within the first hydration layer the results show three preferential orientations for the $\mathrm{OH}$ bond of water molecules: (i) $\mathrm{OH}$ bond points towards the surface $(\cos (\Theta)=-1$ ), (ii) $\mathrm{OH}$ bond remains parallel to the surface $(\cos (\Theta)=0)$, and (iii) $\mathrm{OH}$ bond points to the center of the flow channel $(\cos (\Theta)$ =1). As a conclusion, the results of the MD simulations indicate that flow has no effect on the orientation of water molecules within the first hydration layer on an inert surface, which agrees with the concomitant SHG data. 

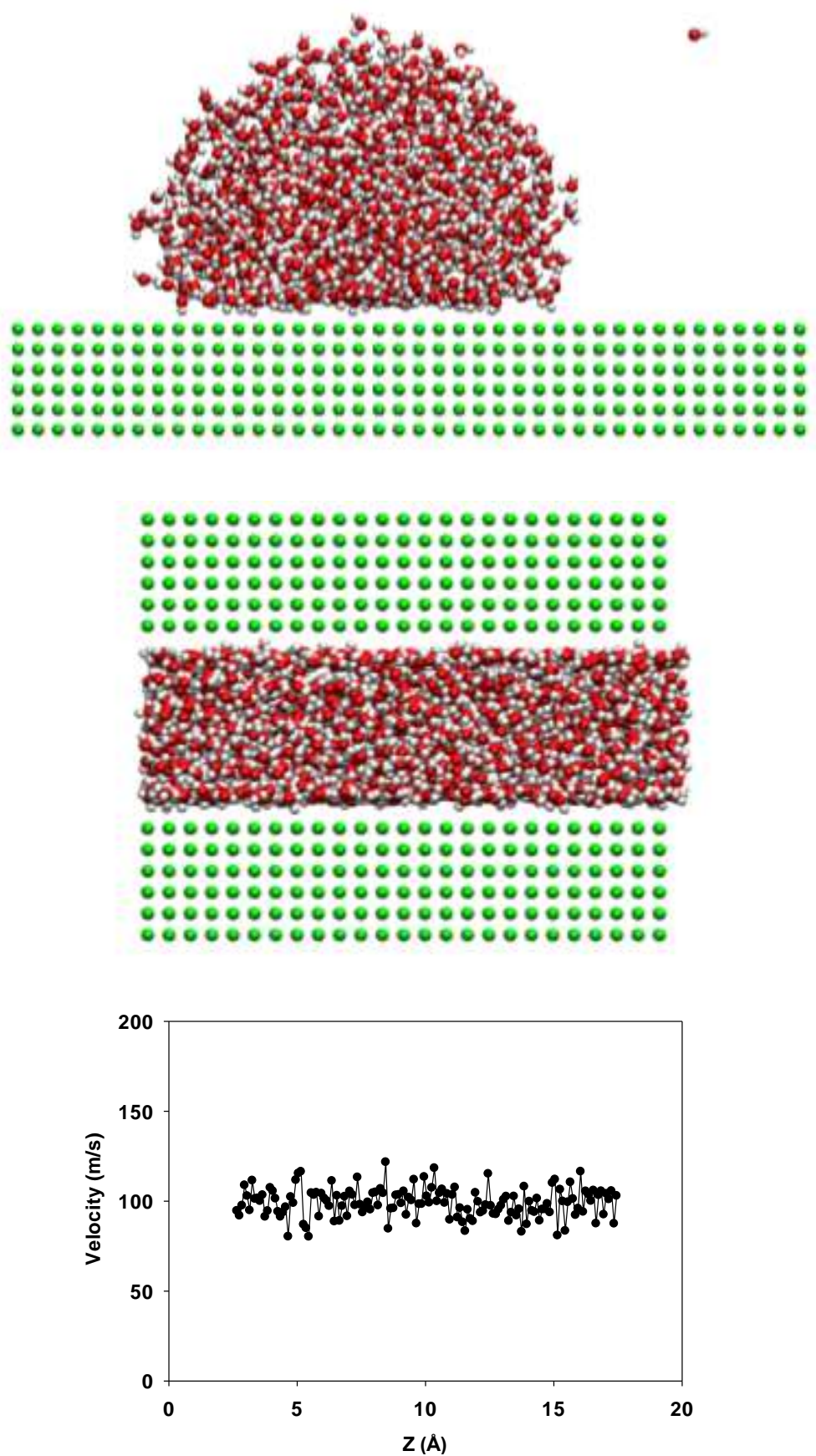

Figure 6. Simulation snapshots representing the systems used for the simulations presented here. Top: A drop of 1000 water molecules is simulated on a hydrophobic surface. Surface area is $8.4 \times 8.4$ $\mathrm{nm}^{2}$. The algorithm replicates the sessile drop experimental procedure. Middle: Simulation set-up to study the effect of flow on the properties of interfacial water. A slit-shaped $2 \mathrm{~nm}$ wide pore is filled with 1632 water molecules. The surface area is $5.25 \times 5.25 \mathrm{~nm}^{2}$.. Bottom: Velocity of water molecules as a function of their distance from the bottom surface within the slit-shaped pore of the middle panel once the flow has been established at steady states. 

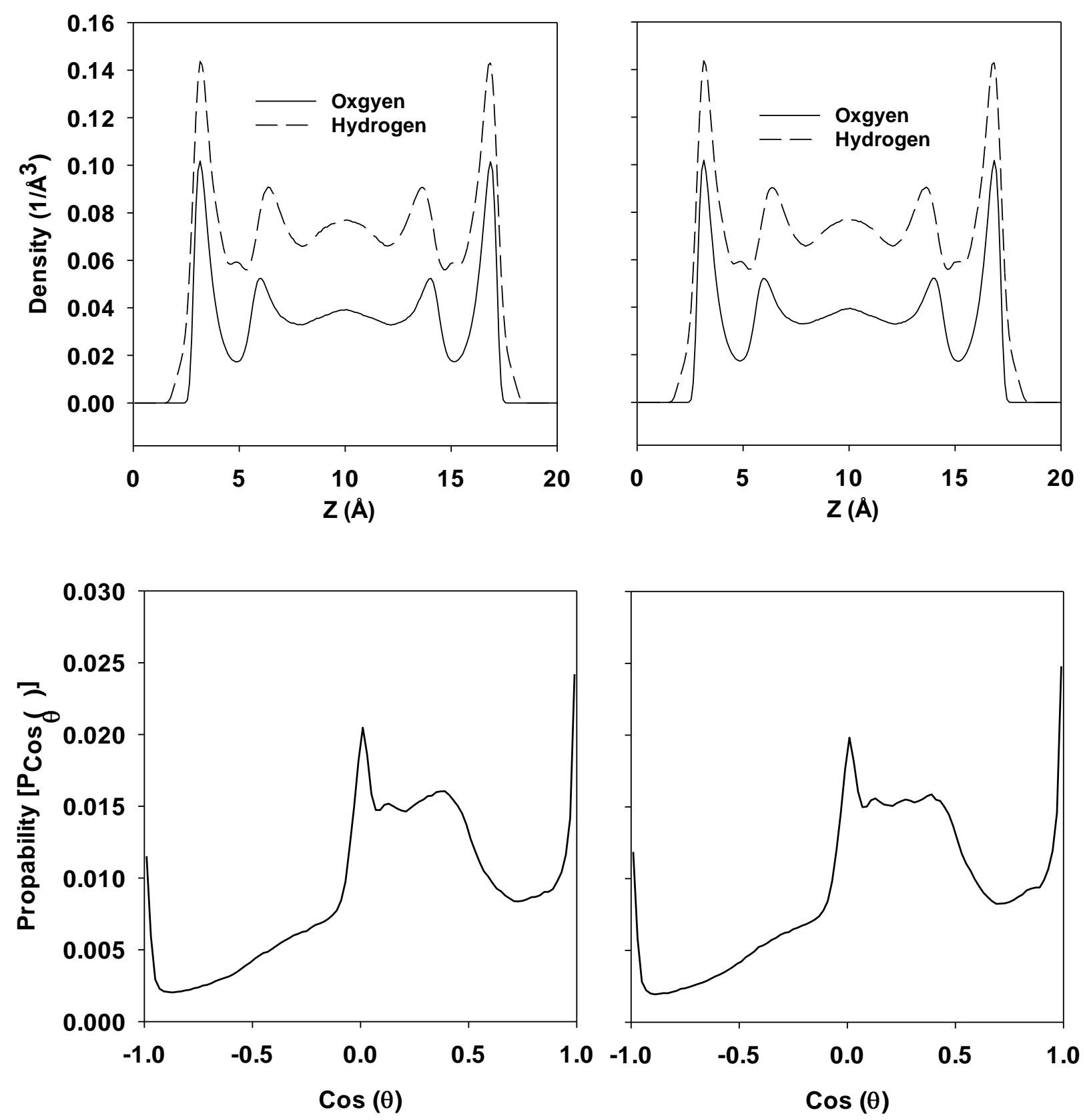

Figure 7. Top: Oxygen (solid) and hydrogen (dash) atomic density profiles for water molecules as a function of position in between the two surfaces within the slit-shaped pore used for the flow simulations (middle panel of Figure 6). Bottom: probability distribution $P_{\cos (\Theta)}$ of the $\cos$ of the angle $\Theta$ formed between the vector normal to surface and the $\mathrm{OH}$ vector of water molecules. Only water molecules found in the first hydration layer (from the surface to first minima in the oxygen density profile) are used for this calculation. For both top and bottom panels the results are shown at equilibrium, when no flow is imposed (left panels) and when the imposed flow is established at steady state (right panels). 


\section{Simultaneous SHG and SP measurements on aged sapphire-c}

The measurements on the aged sapphire-c sample involved fully automated titrations from high to low $\mathrm{pH}$. Streaming potential measurements were conducted simultaneously with the SHG data collection using the cell shown in Figures 1 and 2. One run was carried out under PPP and another under SSP polarization combination. The NLO data sets in the figures discussed in the following sections were scaled to allow better comparison between the PPP and SSP signals.

Figure 8 shows the results of the two titrations. The corrected zeta-potentials show excellent reproducibility (Figure 8a). The IEP of the aged sapphire-c is at $\mathrm{pH} \sim 5.7$, higher than for unaged samples. At $\mathrm{pH}$ values sufficiently far from the IEP, the typical plateauing of the zeta-potential is observed. At very low $\mathrm{pH}$ the increase in ionic strength may explain the slight decrease in zetapotential observed with decreasing $\mathrm{pH}$. The simultaneously obtained SHG data are shown in Figure $8 \mathrm{~b}$ for the two optical configurations. A clear $\mathrm{pH}$-dependence is observed, but interestingly the trends for PPP and SSP differ from each other in the plateau region of the zeta-potential at low $\mathrm{pH}$. The two polarization combinations refer to different structural features. The SSP signal arises from dipole oscillations along the surface normal, i.e. perpendicular to the surface plane. This is the direction in which the electrical double layer evolves. The PPP signal instead, unless measured exactly at the critical angle, is rather a mixture of different contributions. Assuming that deprotonation of so-called in plane hydroxyl groups takes place, the net dipole moment in plane would be predominantly affected, while the deprotonation of out-of-plane hydroxyl groups would primarily affect the dipole moment perpendicular to the surface. The two groups exist on the ideal oxygen terminated sapphire-c surface, but with the aged surface a more detailed discussion in this sense would be hypothetical. 
a)

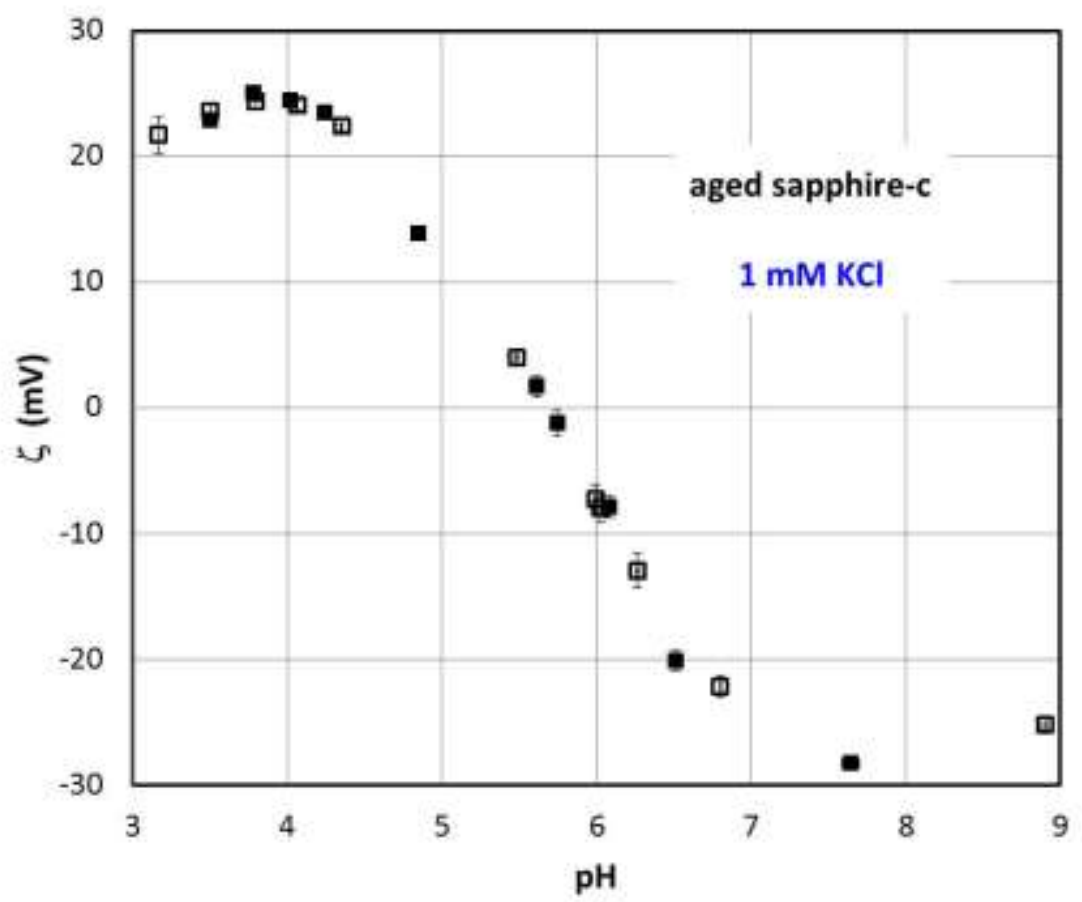

b)

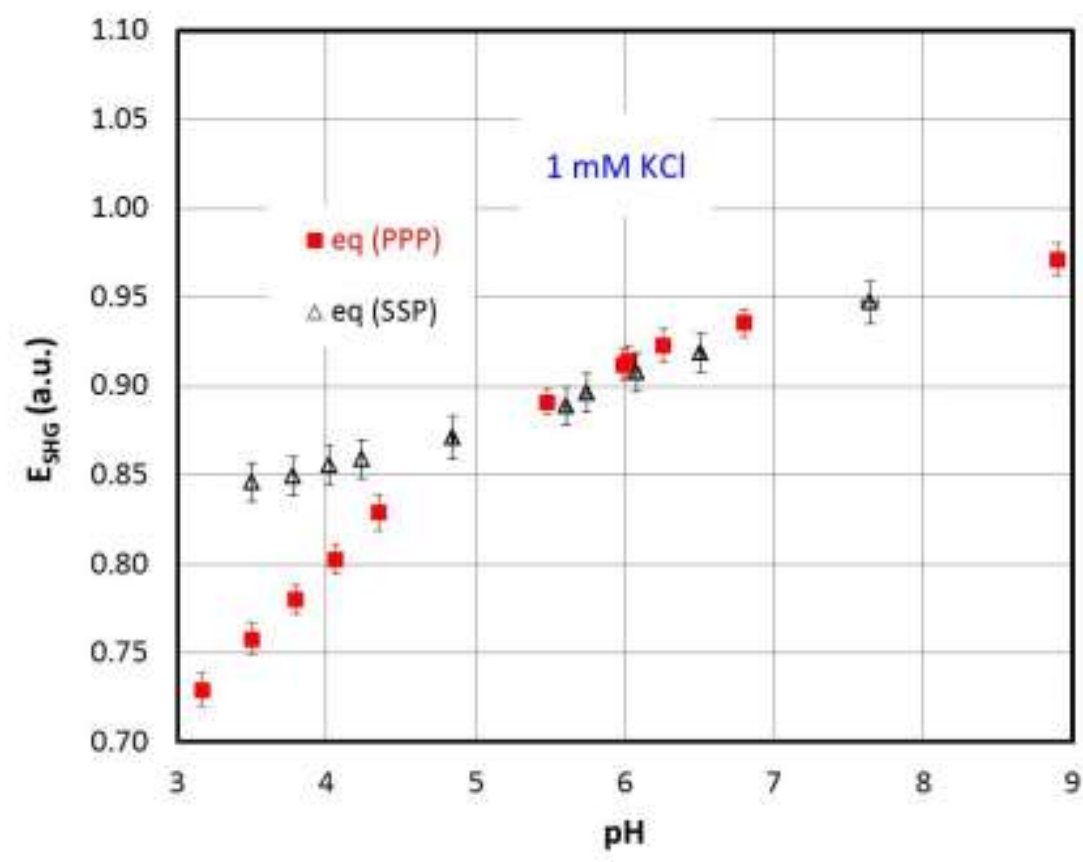

Figure 8: Panel (a): Results from streaming potential measurements using the cell set-up shown in Figure 1 for a continuous $\mathrm{pH}$ titration started at high $\mathrm{pH}>7$ (initial addition of a drop of $\mathrm{KOH}$ ) in 1 $\mathrm{mM} \mathrm{KCl}$. Open and full symbols show individual titrations. The data are corrected for the contribution of the reference surface. Panel (b): Scaled SHG data collected during the two runs of streamingpotential measurements shown in panel a. In one experiment the PPP configuration was implemented, while in the other SSP was used. 
To illustrate directly whether for plateau-situations (Figure 8a), a linear relationship between NLO signals and the zeta-potential as in Figure 5 is still retrieved in Figure 9 the SHG signals and zetapotentials measured simultaneously were plotted versus each other for both polarisations. The results suggest that the SSP signal yields a fairly linear relationship with zeta-potential. For the PPP configuration we observe a deviation from the linear dependence. In fact the NLO signal continues to decrease with decreasing $\mathrm{pH}$, while the zeta-potential levels off. From the shape of the curves, the SSP signal seems to relate to zeta-potentials, while the PPP might perhaps rather represent "inner" surface potentials that are supposed to further increase with decreasing $\mathrm{pH}$. In light of the recent results by Wen et al. [23] the mixing of $\chi^{(2)}$ and $\chi^{(3)}$ contribution depends on the polarization combination, and the relative contribution from the $\chi^{(2)}$ (Stern layer) and from the $\chi^{(3)}$ (diffuse layer) is different in the two cases. Quantification of these aspects cannot be attempted at present, since one of the two incident beams is slightly off the critical angle, and different components of the nonlinear susceptibility tensor will under these conditions contribute to the PPP signal. It is not the aim of the present paper to develop a quantitative description of the NLO signal based on a surface complexation model. This is planned for the near future with more extensive data sets, where the salt level will be varied as well.

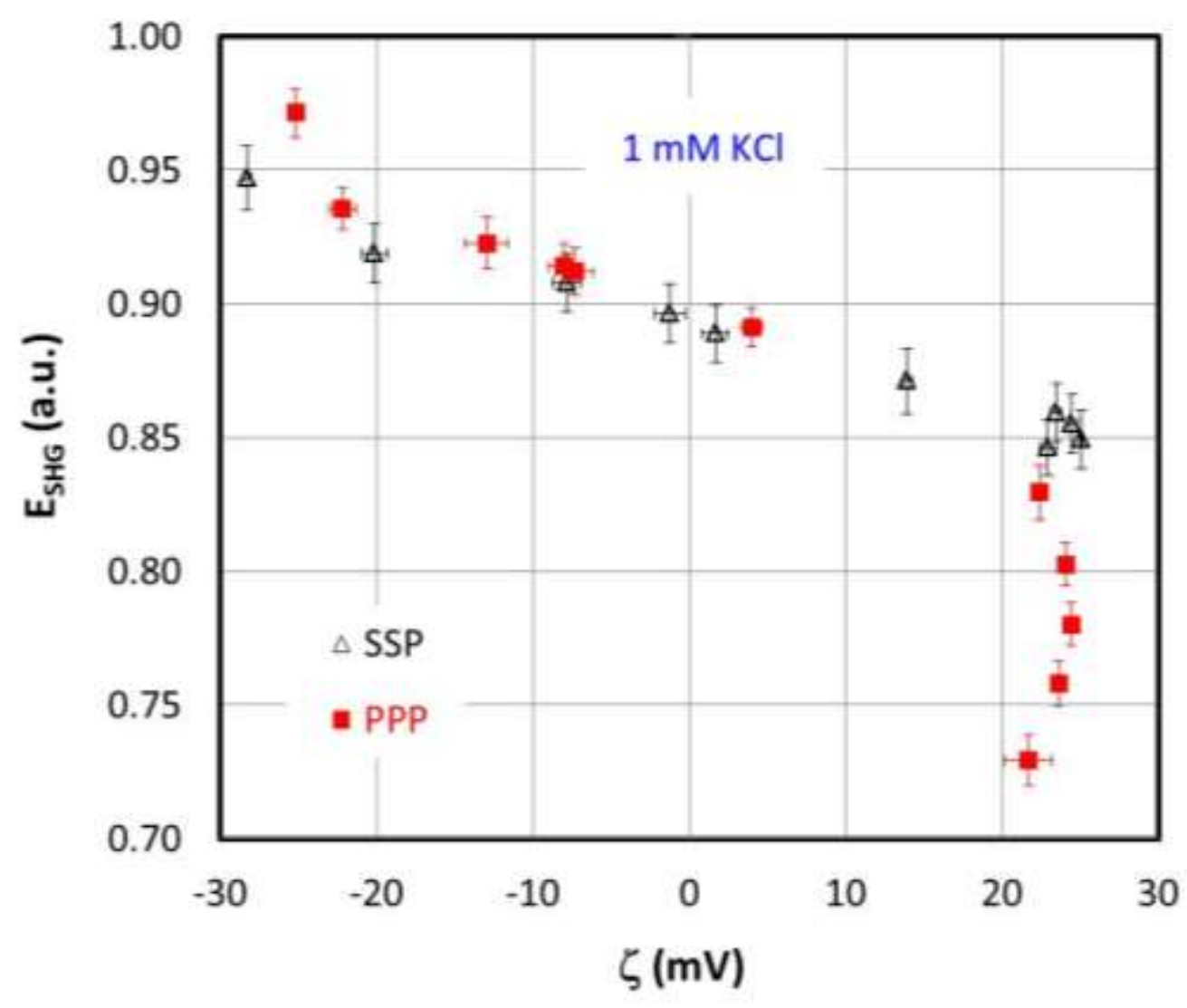

Figure 9. Scaled SHG signal (from Figure 8b) vs. zeta-potential (from Figure 8a) obtained for the aged sapphire-c surface in $1 \mathrm{mM} \mathrm{KCl}$ as a function of $\mathrm{pH}$. 


\section{Conclusions}

\section{Summary of key findings}

Unlike previous experimental observations with a non-equilibrium system no effect of flow on nonlinear optical signals was observed for a hydrophobic and a hydrophilic surface. The results for the hydrophobic surface in this sense agree with the concomitant molecular dynamics simulations, which indicate no change in water structure for no-flow and flow conditions.

The zeta-potentials of two surfaces (hydrophobic and hydrophilic) are proportional to the respective second harmonic generation signal in the vicinity of the isoelectric point. For the hydrophilic surface, depending on the polarization combination in the non-linear optical measurements, such proportionality occurred either over the full $\mathrm{pH}$ range tested (SSP) or showed divergence from the linear behaviour when the zeta-potential achieved the typical plateau (PPP).

\section{Highlight of hypothesis, new concepts and innovations}

Hypothesis: Non-linear optical signals from mineral surfaces have been interpreted in terms of surface potentials. However, the salt dependence of the NLO signals appear to be much more indicative of diffuse layer potentials (which are expected to be close to zeta-potentials). Previous models have used purely diffuse layer approaches to model the NLO signals. However, purely diffuse layer models are known to be inadequate in precisely simulating surface charge (and thus surface potential) of mineral surfaces.

New concept/innovation: A set-up has been tested that allows without ambiguity to measure zetapotential AND second harmonic generation on the same flat surface. The second harmonic results also show the presence or absence of a surface chemical steady state.

\section{Summary of key improvements compared to findings in literature}

Previous measurements of NLO under flow have resulted in surprising observations [27]. The change of the signal under flow compared to no-flow situations in those cases were probably caused by dissolution processes occuring with non-equilibrium solutions, since the effect could be surpressed in equilibrium solutions and with more stable surfaces.

Set-ups for combined measurements of surface and zeta-potential $[44,45]$ or reflectivity and zetapotential [53] on a given surface have been previously used and show the interest in combined experimental data. We are convinced about the usefulness of such data, because they will avoid the ambiguities in the comparisons of separate measurement. In the present work, a set-up is designed that for the first time unambiguously relates macroscopic zeta-potentials to molecular level information concerning interfacial water structure on a flat surface.

\section{Limitations of the new cell}

For the purpose of combined SP/SHG measurements, the cell can in principle accommodate any sample that is suitable for NLO measurements. Ideally the sample is present as a prism or 
hemisphere, large enough to cover the flow channel. Using an optical coupler, it is possible to use any flat surface with appropriate optical properties. The SP method is limited to salt levels below 50 to $100 \mathrm{mM}$, with $100 \mathrm{mM}$ being the upper limit. However, this does not apply to the NLO measurement which can be extended to saturated salt solutions.

\section{Vision for future work}

As alluded to before it might be possible to combine the set-up with a device for "inner" surface potential measurements. Combined streaming and "inner" surface potential measurements (ISFET) have already been performed more than 25 years ago [44, 45]. Alternatively, inclusion of a single crystal electrode [22] in the existing set-up could be attempted.

From the optical point of view an upgrade to sum frequency generation is possible, which would also allow to measure second harmonic and sum frequency generation on a given sample, controlling the identity of the surface through a zeta-potential measurement for example. Finally measuring the reflectivity could allow to obtain direct information about ion-adsorption [52]. This latter technique has also been previously combined with streaming potential measurements [53]. Combination of all these methods has the potential to yield unprecedented experimental data on one solid/solution interface, which will be ultimately required to resolve for example the ambiguities in surface complexation models.

This will help not only in experimentally establishing fundamental relationships between interfacial potentials and non-linear optical properties on IDENTICAL surfaces, but also to overcome the problems related to the overparameterisation of surface complexation modes.

In principle with the use of an external pump, temperature variations can be studied. Although this has not yet been tested, we expect that as along as the cell is not leaking zeta-potentials can be measured as well over a wide range of temperature. The SurPass software has built in temperature corrections

\section{Acknowledgments}

$A A$ is grateful to the German Research Foundation for support (DFG, AB 604/1-1 and AB 604/1-2). Thanks to Dan Lis, Ellen Backus and Mischa Bonn (MPI, Mainz) for making available the fluorite prism. AS is thankful for the generous allocations of computing time provided by the National Energy Research Scientific Computing Center (NERSC) at Lawrence Berkeley National Laboratory, the Oklahoma Center for Education and Research (OSCER), and the University College London High Performance Facility (LEGION@UCL). NERSC is supported by the DOE Office of Science. A.S acknowledges financial support from the European Union via the Marie Curie Career Integration Grant No. 2013-CIG-631435. Financial support from the Deutsche Forschungsgemeinschaft under Grant No. SU 752/2 TRR146 to MS is greatly acknowledged. 


\section{References}

[1] S. Ong, X. Zhao, K.B. Eisenthal, Polarization of water molecules at a charged interface: second harmonic studies of the silica/water interface, Chem Phys Lett 191(3) (1992) 327-335.

[2] A. Abdelmonem, Direct molecular-level characterization of different heterogeneous freezing modes on mica - Part 1, Atmos. Chem. Phys. 17(17) (2017) 10733-10741.

[3] A. Abdelmonem, E.H.G. Backus, N. Hoffmann, M.A. Sánchez, J.D. Cyran, A. Kiselev, M. Bonn, Surface-charge-induced orientation of interfacial water suppresses heterogeneous ice nucleation on $\alpha$-alumina (0001), Atmos. Chem. Phys. 17(12) (2017) 7827-7837.

[4] E. Anim-Danso, Y. Zhang, A. Alizadeh, A. Dhinojwala, Freezing of water next to solid surfaces probed by infrared-visible sum frequency generation spectroscopy, J. Am. Chem. Soc. 135(7) (2013) 2734-2740.

[5] E. Anim-Danso, Y. Zhang, A. Dhinojwala, Surface Charge Affects the Structure of Interfacial Ice, J. Phys. Chem. C 120(7) (2016) 3741-3748.

[6] A.V. Delgado, F. González-Caballero, R.J. Hunter, L.K. Koopal, J. Lyklema, Measurement and interpretation of electrokinetic phenomena, J Colloid Interf Sci 309(2) (2007) 194-224.

[7] J. Luetzenkirchen, T. Preocanin, D. Kovacevic, V. Tomisic, L. Lovgren, N. Kallay, Potentiometric Titrations as a Tool for Surface Charge Determination, Croatica Chemica Acta 85(4) (2012) 391-417. [8] S. Sjöberg, L. Lövgren, The application of potentiometric techniques to study complexation reactions at the mineral/water interface, Aquatic Sciences 55(4) (1993) 324-335.

[9] Y.R. Shen, Surface properties probed by second-harmonic and sum-frequency generation, Nature 337(6207) (1989) 519-525.

[10] Y.R. Shen, V. Ostroverhkov, E.C.Y. Chen, N. Ji, G. Waychunas, Phase-sensitive sum-frequency vibrational spectroscopic studies of water interfaces, Abstr Pap Am Chem S 231 (2006).

[11] K.B. Eisenthal, Second Harmonic Spectroscopy of Aqueous Nano- and Microparticle Interfaces, Chemical Reviews 106(4) (2006) 1462-1477.

[12] K.C. Jena, P.A. Covert, D.K. Hore, The Effect of Salt on the Water Structure at a Charged Solid Surface: Differentiating Second- and Third-order Nonlinear Contributions, The Journal of Physical Chemistry Letters 2(9) (2011) 1056-1061.

[13] A.G.F. de Beer, R.K. Campen, S. Roke, Separating surface structure and surface charge with second-harmonic and sum-frequency scattering, Physical Review B 82(23) (2010) 235431.

[14] G. Gonella, C. Lütgebaucks, A.G.F. de Beer, S. Roke, Second Harmonic and Sum-Frequency Generation from Aqueous Interfaces Is Modulated by Interference, The Journal of Physical Chemistry C 120(17) (2016) 9165-9173.

[15] J. Schaefer, G. Gonella, M. Bonn, E.H.G. Backus, Surface-specific vibrational spectroscopy of the water/silica interface: screening and interference, Phys Chem Chem Phys 19(25) (2017) 16875-16880. [16] X. Zhao, S. Ong, H. Wang, K.B. Eisenthal, New method for determination of surface $\mathrm{pK}_{\mathrm{a}}$ using second harmonic generation, Chem Phys Lett 214(2) (1993) 203-207.

[17] T. Hiemstra, W.H. Van Riemsdijk, On the relationship between charge distribution, surface hydration, and the structure of the interface of metal hydroxides, J Colloid Interf Sci 301(1) (2006) 118.

[18] J.P. Fitts, X.M. Shang, G.W. Flynn, T.F. Heinz, K.B. Eisenthal, Electrostatic surface charge at aqueous/alpha-Al2O3 single-crystal interfaces as probed by optical second-harmonic generation, J Phys Chem B 109(16) (2005) 7981-7986.

[19] G.V. Franks, L. Meagher, The isoelectric points of sapphire crystals and alpha-alumina powder, Colloid Surface A 214(1-3) (2003) 99-110.

[20] J. Lützenkirchen, R. Zimmermann, T. Preočanin, A. Filby, T. Kupcik, D. Küttner, A. Abdelmonem, D. Schild, T. Rabung, M. Plaschke, F. Brandenstein, C. Werner, H. Geckeis, An attempt to explain bimodal behaviour of the sapphire c-plane electrolyte interface, Advances in Colloid and Interface Science 157(1) (2010) 61-74. 
[21] J. Lützenkirchen, G.V. Franks, M. Plaschke, R. Zimmermann, F. Heberling, A. Abdelmonem, G.K. Darbha, D. Schild, A. Filby, P. Eng, J.G. Catalano, J. Rosenqvist, T. Preocanin, T. Aytug, D. Zhang, Y.

Gan, B. Braunschweig, The surface chemistry of sapphire-c: A literature review and a study on various factors influencing its IEP, Advances in Colloid and Interface Science 251 (2018) 1-25.

[22] T. Preočanin, D. Namjesnik, M.A. Brown, J. Lützenkirchen, The relationship between inner surface potential and electrokinetic potential from an experimental and theoretical point of view, Environmental Chemistry 14(5) (2017) 295-309.

[23] Y.C. Wen, S. Zha, X. Liu, S. Yang, P. Guo, G. Shi, H. Fang, Y.R. Shen, C. Tian, Unveiling Microscopic Structures of Charged Water Interfaces by Surface-Specific Vibrational Spectroscopy, Phys Rev Lett 116(1) (2016) 016101.

[24] D.S. Jordan, J.N. Malin, F.M. Geiger, Interactions of Al(III), La(III), Gd(III), and Lu(III) with the Fused Silica/Water Interface Studied by Second Harmonic Generation, Environ Sci Technol 44(15) (2010) 5862-5867.

[25] D.S. Jordan, S.A. Saslow, F.M. Geiger, Exponential Sensitivity and Speciation of Al(III), Sc(III), Y(III), $\mathrm{La}(\mathrm{III})$, and Gd(III) at Fused Silica/Water Interfaces, The Journal of Physical Chemistry A 115(50) (2011) 14438-14445.

[26] A. Selmani, J. Lützenkirchen, N. Kallay, T. Preočanin, Surface and zeta-potentials of silver halide single crystals: $\mathrm{pH}$-dependence in comparison to particle systems, Journal of Physics: Condensed Matter 26(24) (2014) 244104.

[27] D. Lis, E.H.G. Backus, J. Hunger, S.H. Parekh, M. Bonn, Liquid flow along a solid surface reversibly alters interfacial chemistry, Science 344(6188) (2014) 1138-1142.

[28] L.N. Mitchon, J.M. White, Growth and Analysis of Octadecylsiloxane Monolayers on $\mathrm{Al}_{2} \mathrm{O}_{3}(0001)$, Langmuir 22(15) (2006) 6549-6554. A. Ghalgaoui, R. Shimizu, S. Hosseinpour, R. Álvarez-Asencio, C.

McKee, C.M. Johnson, and Mark W. Rutland, Monolayer Study by VSFS: In Situ Response to Compression and Shear in a Contact, Langmuir 30 (11) (2014), 3075-3085.

[29] J. Lützenkirchen, Specific ion effects at two single-crystal planes of sapphire, Langmuir 29(25) (2013) 7726-34.

[30] J. Lützenkirchen, A. Abdelmonem, R. Weerasooriya, F. Heberling, V. Metz, R. Marsac, Adsorption of dissolved aluminum on sapphire-c and kaolinite: implications for points of zero charge of clay minerals, Geochemical Transactions 15(1) (2014) 9.

[31] Y.R. Shen, Optical Second Harmonic Generation at Interfaces, Annu. Rev. Phys. Chem. 40(1) (1989) 327-350.

[32] J.H. Jang, F. Lydiatt, R. Lindsay, S. Baldelli, Quantitative Orientation Analysis by Sum Frequency Generation in the Presence of Near-Resonant Background Signal: Acetonitrile on Rutile $\mathrm{TiO}_{2}(110), \mathrm{J}$. Phys. Chem. A 117(29) (2013) 6288-6302.

[33] Y. Rao, Y.S. Tao, H.F. Wang, Quantitative analysis of orientational order in the molecular monolayer by surface second harmonic generation, J. Chem. Phys. 119(10) (2003) 5226-5236.

[34] X. Zhuang, P.B. Miranda, D. Kim, Y.R. Shen, Mapping molecular orientation and conformation at interfaces by surface nonlinear optics, Phys. Rev. B 59(19) (1999) 12632-12640.

[35] A. Abdelmonem, J. Lützenkirchen, T. Leisner, Probing ice-nucleation processes on the molecular level using second harmonic generation spectroscopy, Atmos. Meas. Tech. 8(8) (2015) 3519-3526.

[36] W.K. Zhang, D.S. Zheng, Y.Y. Xu, H.T. Bian, Y. Guo, H.F. Wang, Reconsideration of secondharmonic generation from isotropic liquid interface: Broken Kleinman symmetry of neat air/water interface from dipolar contribution, The Journal of Chemical Physics 123(22) (2005) 22471301 22471311.

[37] T. Preocanin, A. Selmani, P. Lindqvist-Reis, F. Heberling, N. Kallay, J. Luetzenkirchen, Surface charge at Teflon/aqueous solution of potassium chloride interfaces, Colloid Surface A 412 (2012) 120-128.

[38] J. Lutzenkirchen, T. Preocanin, N. Kallay, A macroscopic water structure based model for describing charging phenomena at inert hydrophobic surfaces in aqueous electrolyte solutions, Phys Chem Chem Phys 10(32) (2008) 4946-4955. 
[39] R. Zimmermann, U. Freudenberg, R. Schweiß, D. Küttner, C. Werner, Hydroxide and hydronium ion adsorption - A survey, Current Opinion in Colloid \& Interface Science 15(3) (2010) 196-202.

[40] T.W. Healy, D.W. Fuerstenau, The isoelectric point/point-of zero-charge of interfaces formed by aqueous solutions and nonpolar solids, liquids, and gases, J Colloid Interf Sci 309(1) (2007) 183-188.

[41] J.P. Fitts, M.L. Machesky, D.J. Wesolowski, X.M. Shang, J.D. Kubicki, G.W. Flynn, T.F. Heinz, K.B. Eisenthal, Second-harmonic generation and theoretical studies of protonation at the water/alpha$\mathrm{TiO}_{2}$ (110) interface, Chem Phys Lett 411(4-6) (2005) 399-403.

[42] L. Bousse, N.F. De Rooij, P. Bergveld, The influence of counter-ion adsorption on the $40 / \mathrm{pH}$ characteristics of insulator surfaces, Surface Science 135(1) (1983) 479-496.

[43] N. Kallay, T. Preocanin, A. Selmani, D. Kovacevic, J. Luetzenkirchen, H. Nakahara, O. Shibata, Thermodynamic Model of Charging the Gas/Water Interface, J Phys Chem C 119(2) (2015) 997-1007. [44] L.J. Bousse, S. Mostarshed, D. Hafeman, Combined measurement of surface potential and zeta potential at insulator/electrolyte interfaces, Sensors and Actuators B: Chemical 10(1) (1992) 67-71. [45] L.J. Bousse, S. Mostarshed, D. Hafeman, Combined measurement of surface potential and zeta potential at insulator/electrolyte interfaces, Solid-State Sensors and Actuators, 1991. Digest of Technical Papers, TRANSDUCERS '91., 1991 International Conference on, 1991, pp. 502-505.

[46] D. Argyris, D.R. Cole, A. Striolo, Hydration structure on crystalline silica substrates, Langmuir 25(14) (2009) 8025-8035.

[47] A. Phan, T.A. Ho, D.R. Cole, A. Striolo, Molecular Structure and Dynamics in Thin Water Films at Metal Oxide Surfaces: Magnesium, Aluminum, and Silicon Oxide Surfaces, The Journal of Physical Chemistry C 116(30) (2012) 15962-15973.

[48] J.A. Ritchie, J.S. Yazdi, D. Bratko, A. Luzar, Metastable sessile nanodroplets on nanopatterned surfaces, The Journal of Physical Chemistry C 116(15) (2012) 8634-8641.

[49] T.A. Ho, D.V. Papavassiliou, L.L. Lee, A. Striolo, Liquid water can slip on a hydrophilic surface, Proceedings of the National Academy of Sciences 108(39) (2011) 16170-16175.

[50] H.J.C. Berendsen, J.R. Grigera, T.P. Straatsma, The missing term in effective pair potentials, The Journal of Physical Chemistry 91(24) (1987) 6269-6271.

[51] T.A. Ho, A. Striolo, Water and methane in shale rocks: Flow pattern effects on fluid transport and pore structure, AIChE Journal 61(9) (2015) 2993-2999.

[52] M. Porus, C. Labbez, P. Maroni, M. Borkovec, Adsorption of monovalent and divalent cations on planar water-silica interfaces studied by optical reflectivity and Monte Carlo simulations, The Journal of Chemical Physics 135 (2011) 064701.

[53] O. Theodoly, L. Cascão-Pereira, V. Bergeron, C. J. Radke, A Combined Streaming-Potential Optical Reflectometer for Studying Adsorption at the Water/Solid Surface, Langmuir 200521 (22), 10127-

10139. 